\title{
Assistive Technology for Promoting Adaptive Behaviors of Children with Cerebral Palsy
}

\author{
Fabrizio Stasolla ${ }^{1^{*}}$, Adele Boccasini ${ }^{2}$, Vincenza Albano ${ }^{3}$ and Annalisa Albano ${ }^{3}$ \\ ${ }^{1}$ Department of Neurosciences, University of Bari, Italy \\ ${ }^{2}$ Lega del Filo d'Oro Research Center, Termini Imerese, Italy \\ ${ }^{3}$ Department of Educational Sciences, Psychology, Communication, University of Bari, Italy
}

\begin{abstract}
Cerebral palsy (CP) refers to a group of non progressive postural and/or movement disturbances dues to defects in the fetus or in the immature brain. It constitutes one of the most frequent cause of developmental disabilities. That is, some of those children are unable to walk, some of them may exhibit postural abnormalities, and some others may present different levels of intellectual disabilities [1-3]. In fact, children with CP rely constantly on parents and/or caregivers assistance and are commonly isolated and passive towards the outside world, with negative consequences on their quality of life [4-5]. Thus, they are unable to acquire new adaptive skills by themselves [6]. To overcome this issue, one may resort on the use of assistive technology (AT) [7-8].
\end{abstract}

AT is an umbrella term including any piece, device and/or equipment aimed at promoting self-determination and independence of participants involved [9]. Therefore, based upon learning principles (i.e. causal association between a behavioral response and environmental consequences) a person with severe to profound developmental disabilities may be enabled with independent access to positive stimulation through the exhibition of small behavioral responses. This latter goal may be pursued through the use of microswitches (i.e. basic forms of AT consisting of electronic devices ensuring autonomously children with multiple disabilities with brief periods of pleasant stimuli) [10]. Although no specific rules exist, some useful guidelines may be outlined for a positive outcome concerning the use of one or more microswitches embedded in an ATbased program. Specifically, a plausible behavioral response should be identified. That is, the response should be already available within the participant's behavioral repertoire and should be performed easily without effort. Subsequently, an adapted microswitch should be selected. Thus, the electronic device (i.e. sensor) should detect the behavioral response and ensure its reliable recording, through a control system unit. Finally, a positive (i.e. high motivating) stimulation to serve as reinforcement should be retained. Therefore, the pleasant stimuli should adequately and sufficiently compensate the response cost. Once the aforementioned conditions are satisfied, the mean frequencies of behavioral responses should reasonably increase during intervention phases if compared to baselines, with beneficial effects for the participants involved [11-12].

Depending upon their levels of functioning, children with $\mathrm{CP}$ may be exposed to different AT-based interventions focused on various rehabilitative objectives. For instance, children disposing of a very low and limited behavioral repertoire combined with severe to profound intellectual disabilities (i.e. motor impairments combined with sensorial and communication disorders), as children with $\mathrm{CP}$ associated to autism spectrum disorders who exhibit multiple disabilities, may be exposed to a single microswitch-based program aimed at enhancing the participant with self-determination and independent access to a unique category of stimulation (e.g. visual stimuli) [13]. Else, a rehabilitative strategy may be planned for fostering choice behaviors with two different behavioral responses and related microswitches for autonomously receiving two categories

\section{Publication History:}

Received: February 09, 2016

Accepted: March 10, 2016

Published: March 12, 2016

\section{Keywords:}

Cerebral palsy, Assistive technology

of stimuli (e.g. visual and auditory stimulation) [14]. Moreover, a combination of microswitch and VOCA (i.e. vocal output communication aid) set-up may be considered crucial as to ensure the participants with the dual goal of independently access to positive reinforcements (i.e. through the microswitch) or asking for social contact with a caregiver (i.e. through the VOCA device) [15]. Additionally, within a dual rehabilitative intervention, a clusterbased technology may be viewed as critical for increasing an adaptive response with the simultaneous reduction of a challenge behavior (e.g. hand/objects mouthing) [16]. Furthermore, as alternative and/or integrative to traditional physiotherapy, microswitches and contingent stimulation may be designed for facilitating both ambulation responses and locomotion fluency [17-18]. For individuals with a higher level of functioning (i.e. motor disorders and mild or moderate intellectual disabilities) one may compare PECS (i.e. picture exchange communication systems) and VOCA protocols (i.e. two different alternative and augmentative strategies), otherwise provide computer-based options finalized at supplying participants with leisure and academic activities or satisfying their personal needs [19]. Finally, children with CP who have extensive motor impairments and who are estimated within the normal range of the intellectual functioning, one may resort on literacy access through the use of combined microswitches and keyboard emulators [20]. Within all the aforementioned programs, indices of happiness and/or indices of positive participations as outcome measure of the quality of life, participants preference checks, and social validation assessment involving sensitive external raters (e.g. parents, teachers, professionals, and/or caregivers) may be carried out as to corroborate the clinical validity of such interventions [21-22].

In light of the above, new research perspectives within this framework should deals with the following topics: (a) extending the suitability and the effectiveness of such AT-based strategies to new

"Corresponding Author: Fabrizio Stasolla, Department of Neurosciences, University of Bari, Piazza Umberto I, 1, 70121 Bari, Italy, E-mail: f.stasolla@psico.uniba.it

Citation: Stasolla F, Boccasini A, Albano V, Albano A (2016) Assistive Technology for Promoting Adaptive Behaviors of Children with Cerebral Palsy. Int J Psychol Behav Anal 2: 110. doi: http://dx.doi.org/10.15344/2455-3867/2016/110

Copyright: (c) 2016 Stasolla et al. This is an open-access article distributed under the terms of the Creative Commons Attribution License, which permits unrestricted use, distribution, and reproduction in any medium, provided the original author and source are credited. 
Citation: Stasolla F, Boccasini A, Albano V, Albano A (2016) Assistive Technology for Promoting Adaptive Behaviors of Children with Cerebral Palsy. Int J Psychol Behav Anal 2: 110. doi: http://dx.doi.org/10.15344/2455-3867/2016/110

participants with developmental disabilities by continuously updating the technology to the participants' and environmental' s needs (i.e. by providing rigorously individualized solutions), (b) enlarging the social validation assessment to new groups of raters, (c) carrying out new maintenance/generalization and/or follow-up phases for substantiating the learning process, and (d) overall positively responding to human, economical and environmental demands.

\section{Competing Interests}

The authors declare that they have no competing interests.

\section{References}

1. Bax MC, Flodmark O, Tydeman C (2007) Definition and classification of cerebral palsy. From syndrome toward disease. Dev Med Child Neuro Suppl 109: 39-41.

2. Bax M, Goldstein M, Rosenbaum P, Leviton A, Paneth N, et al. (2005) Proposed definition and classification of cerebral palsy, April 2005. Dev Med Child Neurol 47: 571-576.

3. Bax MC, Flodmark O, Tydeman C (2007) Definition and classification of cerebral palsy. From syndrome toward disease. Dev Med Child Neurol Suppl 109: 39-41.

4. Rosenbaum P (2011) Family and quality of life: key elements in intervention in children with cerebral palsy. Dev Med Child Neurol 53 Suppl 4: 68-70.

5. Davis E, Davies B, Wolfe R, Raadsveld R, Heine B, et al. (2009) A randomized controlled trial of the impact of therapeutic horse riding on the quality of life, health, and function of children with cerebral palsy. Dev Med Child Neurol 51: 111-119.

6. Rosenbaum PL, Livingston MH, Palisano RJ, Galuppi BE, Russell DJ (2007) Quality of life and health-related quality of life of adolescents with cerebral palsy. Dev Med Child Neurol 49: 516-521.

7. Lancioni GE, Singh NN. Assistive echnologies for people with diverse abilities. 2014. New York: Springer.

8. Lancioni GE, Sigafoos J, O'Reilly MF, Singh NN. Assistive Technology. Interventions for Individuals with Severe/Profound and Multiple Disabilities. 2012. New York: Springer.

9. Stasolla F (2015) Assistive Technologies and Microswitches for Promoting Constructive Engagement by Children with Developmental Disabilities. Int J Behav Res Psychol 3: 1-2.

10. Stasolla F (2015)Assistive Technology for Children with Multiple Disabilities J Psychol Psychoanal 1:001e.

11. Lancioni GE, O'Reilly MF, Singh NN, Stasolla F, Manfredi F, et al. (2004) Adapting a grid into a microswitch to suit simple hand movements of a child with profound multiple disabilities. Percept Mot Skills 99: 724-728.

12. Stasolla F, De Pace C (2014) Assistive technology to promote leisure and constructive engagement by two boys emerged from a minimal conscious state. NeuroRehabilitation 35: 253-259.

13. Lancioni GE, O'Reilly MF, Singh NN, Sigafoos J, Chiapparino C, et al. (2007) Enabling a young man with minimal motor behavior to manage independently his leisure television engagement. Percept Mot Skills 105 47-54.

14. Stasolla F, Damiani R, Caffò AO (2014) Promoting constructive engagement by two boys with autism spectrum disorders and high functioning through behavioral interventions. Research in Autism Spectrum Disorders 8: 376 380 .

15. Lancioni GE, Bosco A, Belardinelli MO, Singh NN, O'Reilly MF, et al. (2014) Technology-based intervention programs to promote stimulation contro and communication in post-coma persons with different levels of disability. Frontiers in Human Neuroscience 8: 48.

16. Stasolla F, Perilli V, Damiani R, Caffò AO, Di Leone A, et al. () A microswitchcluster program to enhance object manipulation and to reduce hand mouthing by three boys with autism spectrum disorders and intellectual disabilities. Research in Autism Spectrum Disorders 8: 1071-1078.

17. Stasolla F, Caffò AO (2013) Promoting adaptive behaviors by two girls with Rett syndrome through a microswitch-based program. Research in Autism Spectrum Disorders 7: 1265-1272.
18. Lancioni GE, Singh NN, O'Reilly MF, Sigafoos J, Oliva D, et al. (2010) Promoting ambulation responses among children with multiple disabilities through walkers and microswitches with contingent stimuli. Res Dev Disabil 31:811-816.

19. Stasolla F, Caffò AO, Picucci L, Bosco A (2013) Assistive technology for promoting choice behaviors in three children with cerebral palsy and severe communication impairments. Res Dev Disabil 34: 2694-2700.

20. Chiapparino C, Stasolla F, De Pace C, Lancioni GE (2011) A touch pad and a scanning keyboard emulator to facilitate writing by a woman with extensive motor disability. Life Span Disabil 14: 45-54.

21. Stasolla F, Caffò AO, Damiani R, Perilli V, Di Leone A, et al. (2015) Assistive technology-based programs to promote communication and leisure activities by three children emerged from a minimal conscious state. Cogn Process 16: 69-78.

22. Lancioni GE, O'Reilly MF, Singh NN, Groeneweg J, Bosco A, et al. (2006) A social validation assessment of microswitch-based programs for persons with multiple disabilities employing teacher trainees and parents as raters. Journal of Developmental and Physical Disabilities18: 383-391. 